\title{
Strongly magnetic, rapidly rotating main-sequence stars
}

\section{Keith William Robson}

The purpose of this thesis is to study the effects of uniform rotation and strong dipolar magnetic fields, on the structure and properties of main-sequence stars using a perturbation method.

The method proceeds by constructing a spherical star which takes into account exactly the spherically symmetric part of the perturbing forces. The non-spherical terms are then treated as a perturbation about this spherical model. This method is described in some detail in Chapter 1 where it is used to construct upper main-sequence stellar models. The main assumptions used are zero meridional circulation, the coincidence of the magnetic and rotation axes, and that the convection in the core has no effect on the magnetic field. When the perturbing forces are weak, so the results could be compared to other theoretical studies, good agreement is found. However, with strong perturbing forces, considerable alterations to the stellar models occur.

From the results of Chapter 1, the gross characteristics of total luminosity, mean effective surface temperature and mean radius are calculated in Chapter 2 for a number of stellar masses, with various strengths of the perturbing forces. From these calculations, H-R diagrams are constructed which verify the trends of other authors for the case of strong rotation, and also show that strongly magnetic stars are likely to lie below the main-sequence. Also, the perturbed surfaces of zero pressure are calculated in Chapter 2, and it is shown that the surface of a strongly magnetic star approaches a doughnut shape, and lies wholly within the surface of a star without a magnetic field.

Received 29 May 1972. Thesis submitted to Monash University, January 1972. Degree approved, May 1972. Supervisor: Dr J.J. Monaghan.

313 
In Chapter 3, other physical characteristics are calculated for the various upper main-sequence stellar models including the magnetic, rotational, thermal and gravitational energy, from which checks on the virial theorem are made. Also the condition for dynamical stability and the upper limit of the root-mean-square field given by the virial theorem are checked for the strongly magnetic models. Although many of the models constructed have mean internal magnetic fields much greater than the observed surface fields, it is shown in Chapter 3 that with sufficient rotation, a star with a huge internal magnetic field appears to have only a moderate field, or even be visibly non-magnetic.

In Chapter 4 a grey atmosphere is placed on the upper main-sequence stellar models, and by calculating the local surface gravity and hence the local effective surface temperature the observational parameters are determined for rotating stars with and without strong magnetic fields at various aspect angles. In these calculations allowance is made for the fact that observing strongly magnetic stars equator on, not all its surface can be seen. A colour-magnitude diagram is constructed, which gives the pole on and equator on positions of stars with various magnetic field strengths. Whilst confirming trends of previous authors for the case of raptd rotation with negligible magnetic field strength, Chapter 4 shows that considerable alterations in the observable characteristics occur with the inclusion of a strongly magnetic field.

As a further application of the perturbation technique the structure and properties of rotating, strongly magnetic lower main-sequence stars are studied in Chapter 5. Most of the calculations made in Chapters 1,2 , and 3 are repeated for these cooler stars. However, the effect of convection on the magnetic field is treated by a phenomenological model. This model simulates the outer convective layer pushing the magnetic field towards the centre so reducing the surface field. The H-R diagram shows a movement to the left of the main-sequence for strongly magnetic stars. 